\title{
Governance of the International Linear Collider Project
}

\section{B. Foster ${ }^{1}$}

University of Oxford

Department of Physics, Denys Wilkinson Building, Keble Road, Oxford, OX1 3RH, UK

E-mail: b. foster@physics.ox.ac.uk

B. Barish

California Institute of Technology, USA

J.-P. Delahaye,

CERN, Switzerland

U. Dosselli

INFN, Italy

E. Elsen

DESY, Germany

M. Harrison

Brookhaven National Laboratory, USA

J. Mnich

DESY, Germany

\section{J.M. Paterson}

SLAC National Accelerator Laboratory, USA

F. Richard

LAL, Orsay, France

S. Stapnes

CERN, Switzerland

\section{A. Suzuki}

KEK, Japan

G. Wormser

LAL, Orsay, France

S. Yamada

KEK, Japan

$1 \quad$ Speaker 
Governance models for the International Linear Collider Project are examined in the light of experience from similar international projects around the world. Recommendations for one path which could be followed to realise the ILC successfully are outlined.

35th International Conference of High Energy Physics (ICHEP2010) 


\section{Overview}

The International Linear Collider (ILC) is a unique endeavour in particle physics; fully international from the outset, it has no "host laboratory" to provide infrastructure and support. The realization of this project therefore presents unique challenges, in scientific, technical and political arenas. This document outlines the main questions that need to be answered if the ILC is to become a reality. It describes the methodology used to harness the wisdom displayed and lessons learned from current and previous large international projects. From this basis, it suggests both general principles and outlines a specific model to realize the ILC. It recognizes that there is no unique model for such a laboratory and that there are often several solutions to a particular problem. Nevertheless it proposes concrete solutions that the authors believe are currently the best choices in order to stimulate discussion and catalyze proposals as to how to bring the ILC project to fruition. The ILC Laboratory would be set up by international treaty and be governed by a strong Council to whom a Director General and an associated Directorate would report. Council would empower the Director General to give strong management to the project. It would take its decisions in a timely manner, giving appropriate weight to the financial contributions of the member states. The ILC Laboratory would be set up for a fixed term, capable of extension by agreement of all the partners. The construction of the machine would be based on a Work Breakdown Structure and value engineering and would have a common cash fund sufficiently large to allow the management flexibility to optimize the project's construction. Appropriate contingency, clearly apportioned at both a national and global level, is essential if the project is to be realised. Finally, models for running costs and decommissioning at the conclusion of the ILC project are proposed.

This document represents an interim report of the bodies and individuals studying these questions inside the structure set up and supervised by the International Committee for Future Accelerators (ICFA). It represents a request for comment to the international community in all relevant disciplines, scientific, technical and most importantly, political. Many areas require further study and some, in particular the site selection process, have not yet progressed sufficiently to be addressed in detail in this document. Discussion raised by this document will be vital in framing the final proposals due to be published in 2012 in the Technical Design Report being prepared by the Global Design Effort of the ILC.

\section{Introduction}

In the early 2000's several study reports [1] were issued by Asian, European and American regional bodies, representing the relevant high-energy physics communities, on possible organisational structures for the project management of a Linear Collider (LC). The Consultative Group on High- Energy Physics of the Organisation for Economic Cooperation and Development (OECD) also issued a report [2] on their consensus, concurrently with these regional reports. All reports agreed that a highenergy electron-positron LC is the next major facility on the world high-energy physics 
roadmap. Together, these reports laid the foundations for a world organisation for the design development of an LC, leading to establishment of the Global Design Effort (GDE) for the International Linear Collider.

These regional and international reports systematically identified most of the organisational, legal, budgetary and political issues associated with the actual construction and management of the ILC project. The GDE is presently engaged in producing a Technical Design of the ILC Project before the end of 2012, when it will be presented to the community and interested government agencies. Therefore, we aim to produce recommendations agreed by the international research community for the Organisation or Governance of the ILC Project on a similar timescale,.

The purpose of this document, which we classify as a "request for comments" is to facilitate a structured discussion on these governance issues of the International Linear Collider in the construction and operation stage, by presenting a possible solution model and outlining other options, and by inviting inputs from the members of relevant international research communities. It should be emphasized that the solution presented below is not the only one possible; others could no doubt be made to work. Nevertheless, in the opinion of the study groups, it is the preferred option.

Several key observations are worth noting at this point.

a. Consensus-based Guidelines for Global Projects need to be developed - the community of high-energy physics, under ICFA, has developed "ICFA Guidelines for the Interregional Utilization of Major Regional Experimental Facilities for High-Energy Physics Research" [3], which have been successful as guidance for the execution of international research programs at large accelerator facilities. However, the ILC is likely to be outside the scope of the existing guidelines, since major portions of construction and operation budgets are expected to be internationally, rather than regionally or nationally, shared.

Therefore, it is intended to annotate the present ICFA Guidelines by adding statements on "Global" projects, in which major parts of the construction and operation budgets are shared. An attempt should be made to clarify the separation, connection and coexistence between the ILC-like and non-ILC-like (and, consequently, more traditional) international projects.

b. A Linear Collider will not be the only project to be pursued by the world high-energy physics community. Besides the LC, a strong need could emerge for new, next generation international accelerator facilities, which require existing laboratories to lead the related research, design and development efforts. Some such projects might be similar to the ILC in terms of the globally distributed nature of resources, and some may be more localized to specific regions. In both cases, the co-existence of individual institutions with the project and the sharing of local/regional personnel and facilities will require careful analysis. Therefore, the ILC laboratory organisation will make provision for evolutionary and continuous involvement of existing national/regional research institutions. 
c. The separation of technical/scientific and political aspects. Without doubt, the final negotiations and decisions concerning the legal agreements, budget sharing and site selection for the ILC will have to be made by the appropriate government agencies of the interested nations/regions. On the other hand, the technical context and resultant boundary conditions or specifications for the project (such as the base performance parameters or the technical specifications for possible sites) should be dictated by the scientific merits. This aspect should be insulated from political considerations.

Therefore, it would be advantageous to identify "where the scientists end, and where the government officers and statesmen begin", and develop our analysis accordingly. A notable example is the issue of site selection, where the critical requirement is that this process converges to a site truly suitable for construction and operation of the ILC from the technical and scientific viewpoints. Here, we propose that the members of the academic sector play leadership roles in defining the specifications, while the political sector makes the best executive judgment among the candidate sites that are known to satisfy the previously established technical specifications. We are continuing to study various examples of the site selection process, both in similar projects, such as the European Spallation Source (ESS) and ITER, which had a long and difficult site selection, as well as in other fields, such as the Olympic Games, where the process runs much more smoothly.

We recommend that the International Linear Collider Steering Committee (ILCSC) leads the effort to establish such site-selection criteria. These site criteria and a subsequent validation process of prospective sites from the technical standpoint, will establish a set of site candidates all of which are verified as satisfying the scientific and technical site requirements for the ILC. Subsequent government-level negotiations of these "pre-validated" site candidates will result in a site that is technical suitable for the ILC.

A large number of more technical issues, in particular intellectual property rights and ownership, are not addressed in this document but deferred until the final report. It should be noted that ITER has made an extensive study of IP issues that can serve as a reference for the ILC.

In the following sections, more specific analyses and a possible organisational model for the ILC and its time-evolution are presented.

\section{ILC Governance}

\subsection{Introduction}

The International Linear Collider (ILC) is in many ways a unique project, certainly in particle physics. It is the first accelerator to be proposed on a truly international basis, having no initial major "host" laboratory in which the project could be incubated. It was set up from the outset in a fully international way with all countries and regions on an equal basis. The construction of the ILC will require a 
major political effort from governments around the world; its governance will necessarily be complex and requires a consensus across different national systems and traditions that will not be easy to forge. This section is an interim report of an effort inside the Global Design Effort of the ILC. It attempts to clarify issues and recommend possible ways forward. Its aim is to help governments to identify the major issues that must be addressed if an ILC laboratory is to be fit for purpose and capable of both building the accelerator and carrying out the exciting physics programme of the ILC. The ILC organisation proposed is limited to building and carrying out the physics programme of the ILC and will be dissolved when its mission is completed.

\subsection{Working methods}

Although the ILC is unprecedented in particle physics, a great deal of experience has been built up on the governance of other projects of substantial size and wide international involvement. However, perhaps only ITER is really comparable in both these aspects and even then there are substantial differences. This means that it is impossible simply to take over wholesale prescriptions that worked well in previous particle physics projects or in the current generation of large international facilities. This does not imply that lessons cannot be learnt from them; on the contrary, the approach of the authors has been systematically to investigate the Governance arrangements for many international projects and to organise the data obtained into a series of pro formas. By distilling the information into common headings, useful comparisons could be made and lessons drawn. In addition to reading the proposal and other documents produced by these projects, several meetings and discussions have been held with senior members of many of the projects, in which experience and ideas have been exchanged and refined.

The projects that have been investigated are: ALMA; ESS; FAIR; ITER; SKA and XFEL. Both ESS and SKA are at an early stage of development and therefore do not necessarily have fixed proposals for governance; nevertheless we have examined and discussed what is available and incorporated it into our considerations where appropriate. There are many similarities between FAIR and XFEL; we have concentrated on the proposals for XFEL but where FAIR has differing features they have been taken explicitly into consideration.

The information on the projects was organised wherever possible into a common format in pro formas. The headings under which information was organised were:
a. Legal Status
b. Management Structure
c. Representation and voting structure in governing body
d. Duration of agreement
e. Attribution of in-kind contributions, value engineering, etc.
f. Running costs \& decommissioning
g. Budgetary control 
The pro formas were discussed and evaluated in several meetings of the ILC working groups in order to reach a series of recommendations for each of the sections of the pro formas. These are outlined below, where bold sections represent the recommendations and the italic text below is a commentary on that recommendation, adducing reasons and observations on the conclusion. It should be emphasized that, while there were often strong reasons to reach a particular recommendation, there were also sometimes several possible conclusions with no strong preference for any particular one; the recommendation of a particular path does not mean that other choices would not work.

\section{Recommendations}

\subsection{Legal Status}

ILC should be set up as an international treaty organisation similar to ITER, taking advantage of zero VAT rating and similar privileges.

Projects examined include three currently utilized models for legal status: no legal entity, limited company and treaty organisation. The group strongly recommends that ILC must have its own legal identity. Experience from XFEL and FAIR implies that the foundation of a limited-liability company is no easier, quicker or less complex than a treaty organisation. A treaty organisation with a finite duration is stronger and more flexible than a limited company. A vitally important part of the treaty is to guarantee access to the ILC laboratory to all interested parties. The circumstances of the US with regard to treaties can be accommodated by the same arrangement as used for ITER. Participation of individual countries in the ILC can be through regional organisations, e.g. CERN, and use can be made of existing research infrastructure frameworks, e.g. ERIC in the European Union, where appropriate. While preferring a treaty, both that and a limited company could be made to work.

\subsection{Management Structure}

ILC should have a strong Council as the ultimate governance body. Council delegates should be of sufficient standing to make decisions in a timely fashion. The ILC should have a Director General and a Directorate, proposed for Council ratification by the DG. The DG should have significant delegated authority from the Council, allowing him or her to act decisively without continual need to refer back to Council.

All projects examined have Councils representing the member states; some are stronger than others. Council should meet at least twice a year. It is essential that a DG should be appointed in whom Council has confidence and whom it trusts to manage the laboratory and project. He or she must have suitable delegated authority to keep the project on track. The level of delegated authority in, for example, ITER does not seem to us sufficient to manage the project optimally. 
4.3 Representation and voting structure in governing body

Each Council member state ${ }^{1}$ should have 2 official delegates and a maximum of 2 advisors. One of the two delegates should be a particle physicist. There should be the option, every few years, of Ministerial Council Meetings in which delegates are the relevant government minister.

Council should decide questions not of a financial nature by simple majority; financial questions should be decided by a qualified majority voting decided by a majority of financial contributions plus a majority of individual member states.

It seems important to keep the numbers attending Council meetings as small as possible, consistent with ensuring that each member state has a delegate representing the government and another to give a scientific perspective on the work of the ILC laboratory. This recommendation is again modelled on the CERN experience. CERN Council does not explicitly have Ministerial sessions in contrast to e.g. ESA. Appropriate Ministerial involvement with the organisation is important when major strategic decisions are required. The option of having such meetings on a regular basis is important.

Most of the projects examined have a weighted voting system, with a tendency to reach decisions by requiring a higher weight of financial contributions than required at the CERN Finance Committee. It is unnecessary to specify the details of the voting system here but the pattern of a majority of financial contributions and/or a majority of members seems a good one.

\subsection{Duration of ILC agreement}

The ILC agreement should be fixed term - a construction period of $\sim 9$ years plus 20 years of operation; it should be extendable by agreement of Council in periods of 5 years. Withdrawal would not be allowed until a minimum of 10 years after the agreement comes into force and then only after 1 full year after notice of withdrawal.

All projects have a fixed term which can be extended after agreement by all members. The construction period represents the current best guess from the ILC Civil Construction experts including necessary initial tool-up time after the construction agreement is signed. The physics programme of the ILC would extend over at least 20 years and would include an energy upgrade to $1 \mathrm{TeV}$ as well as possible technology changes to reach even higher energies. It is essential for international organisations to have stability of membership in order to plan sensibly; hence withdrawal should be inhibited by considerable barriers.

\footnotetext{
1 The definition of a "member state" should be as flexible as possible and include groupings of nations represented by a coordinating body, for example CERN or JINR.
} 
4.5 Attribution of in-kind contributions, value engineering, etc.

The ILC construction project should be based on a Work Breakdown Structure (WBS) system. In-kind contributions will be likely to form the majority of contributions to the project's infrastructure. An agreed register of WBS items should be set up and a committee constituted to consider bids for WBS items from member states. Value engineering should be used in defining the "value" of each WBS item. There should be an adequate Common Fund (of at least $20 \%$ ) in order to give management enough flexibility. There should be no strict "juste retour".

Use of WBS is now standard in all major projects, as is value engineering, which is used to optimise the performance/cost ratio and thereby determine the size of financial contributions attributed to WBS items. The value estimate must be based on the closeto-final design and the industrial procurement model, including the likely number of qualified vendors. The committee to adjudicate award of WBS items will need optimally to match the expertise and track-record of the bidding manufacturer, the financial contribution of the member state and the requirements of the project. While "juste retour" would ensure that all member states get a return of funding to their industries proportional to their input, this is not recommended as it tends to increase the cost of the project and can also result in the project being unable to select the best vendor for the job. It is vital that the project has a sufficiently large Common Fund so that the management can react to problems in a flexible way and allocate additional resources to time-critical areas of the project. Experience from for example ITER implies that a Common Fund larger than the 12\% allocated for ITER is required. We believe that 20\% is a reasonable minimum Common Fund of a project of the size and complexity of the ILC. If the Common Fund is too small, schedule slippage and concomitant cost increases and inefficiency are highly likely.

\subsection{Contingency}

If and when needed, the Council should have the authority to call on a central contingency budget with a maximum of $10 \%$ of the total project cost and to allocate it as appropriate. Increases in costs to produce a WBS item smaller than $\mathbf{2 5 \%}$ or some other agreed ceiling in cash should be borne by the country with responsibility for that item; they are recommended to have appropriate internal contingency. It is important to avoid double counting between the central contingency and a country's internal contingency in arriving at the overall project costing. If costs for a WBS item increase beyond the agreed ceiling, the case could be referred to and considered by a standing Board and either referred back to the submitting country or referred to Council for release of central contingency, as appropriate.

Exhaustion of the central contingency should lead to appropriate descoping of the project to be decided by management with Council's agreement.

Generally speaking, the provision of in-kind contributions carries with it a responsibility for the contracting member state to bear any cost overruns incurred in providing the WBS item. However, it is likely that some items may well incur cost 
increases because of factors beyond the control of the provider, or involving some other exceptional factor. An example would be if the growth in cost were substantially related to design changes in other areas of the project. Experience with other projects teaches that it is necessary to deal flexibly with increases in cost for particular WBS items. The expectation should be that countries assigned a WBS item should normally be responsible for any cost increases incurred in providing it; however, it is necessary to recognize that there may be exceptional circumstances where this is not appropriate and to put in place mechanisms to adjudicate such cases. The size of the central contingency proposed is in line with that adopted by other large projects.

Recent experience in budgetary growth for large international projects has not been encouraging. In order to reassure governments that the ILC project, once approved, will not spiral into major cost overruns, we believe that it is necessary to give assurances that descoping the project is possible in order to contain costs if this is the decision of the ILC Council. The ILC, being highly modular, can be descoped in a way which is not straight-forward for a project such as for example ITER. The most obvious method of descoping is to reduce the energy reach of the machine by installing fewer superconducting cavities.

\subsection{Running costs and decommissioning}

Running costs should be evaluated at the time of setting up the organisation and a suitable algorithm agreed to. A commonly chosen algorithm is that running costs should be distributed roughly proportional to capital contributions.

Decommissioning should be the responsibility of the state that provided that WBS item; the Host State should have residual responsibility.

Both of these recommendations follow common practice of most of the projects that we have examined.

\section{References}

[1] G. Kalmus et al., ECFA-2003-225, CERN, Geneva, (2003); http://cdsweb.cern.ch/record/905067/files/ecfa-2005-225.pdf S. Yamada et al; Report of the JLC Globalisation Committee, KEK, Japan (2002) http://lcdev.kek.jp/GLCC/glcc report.pdf

[2] OECD Global Science Forum: Report of the Consultative Group on High Energy Physics, Paris, France (2002); http://www.oecd.org/dataoecd/2/32/1944269.pdf

[3] http://www.fnal.gov/directorate/icfa/icfa_guidelines.html 